\title{
Lung innervation in the eye of a cytokine storm: neuroimmune interactions and COVID-19
}

\section{Francesco De Virgiliis and Simone Di Giovanni iD}

Abstract |COVID-19 is an infectious disease caused by the coronavirus SARS-CoV-2, which was first reported in Wuhan, China, in December 2019 and has caused a global pandemic. Acute respiratory distress syndrome (ARDS) is a common feature of severe forms of COVID-19 and can lead to respiratory failure, especially in older individuals. The increasing recognition of the neurotropic potential of SARS-CoV-2 has sparked interest in the role of the nervous system in respiratory failure in people with COVID-19. However, the neuroimmune interactions in the lung in the context of ARDS are poorly understood. In this Perspectives article, we propose the concept of the neuroimmune unit as a critical determinant of lung function in the context of COVID-19, inflammatory conditions and ageing, focusing particularly on the involvement of the vagus nerve. We discuss approaches such as neurostimulation and pharmacological neuromodulation to reduce tissue inflammation with the aim of preventing respiratory failure.

Coronaviruses are important pathogens for humans and animals and are usually associated with respiratory and gastrointestinal infections. Most human coronaviruses, such as hCoV-229E, OC43, NL63 and HKU1, cause mild respiratory diseases. However, the worldwide spread of two previously unrecognized viruses, the severe acute respiratory syndrome coronavirus (SARS-CoV) and Middle East respiratory syndrome coronavirus (MERS-CoV), revealed to the world the lethal potential of human coronaviruses ${ }^{1}$. Another highly pathogenic coronavirus, which has been named SARS-CoV-2 and causes a disease known as COVID-19, was reported in December 2019 in Wuhan, China ${ }^{2}$. This novel virus caused a national outbreak of severe pneumonia in China and has rapidly spread around the world owing to its high infectivity, causing a global pandemic.

SARS-CoV-2 can cause acute, highly lethal pneumonia with clinical symptoms similar to those reported for SARS-CoV and MERS-CoV ${ }^{1}$. A substantial proportion of patients who are admitted to intensive care units worsen in a short period of time and die from respiratory failure ${ }^{1}$.
Importantly, coronavirus infections are not always confined to the respiratory tract, and clinical studies have revealed a degree of symptomatic heterogeneity in people with COVID-19, with anosmia being a frequent occurrence $^{3}$ and severe neurological symptoms being reported in some cases ${ }^{4-7}$. These observations suggest that a range of different tissues and cell types, including the nervous system ${ }^{3,8}$, are affected by the virus.

The entry of SARS-CoV-2 into human host cells is mediated mainly by the transmembrane proteins angiotensin-converting enzyme 2 (ACE2) and transmembrane protease serine 2 (TMPRSS2), which are highly expressed in the airway epithelium, lung parenchyma, vascular endothelium, heart, kidney and small intestine in humans ${ }^{9-11}$. Most notably, both ACE2 and TMPRSS2 are expressed in the olfactory epithelium and lower respiratory tract, which are densely innervated ${ }^{12,13}$. These barrier tissues are postulated to be key invasion sites for SARS-CoV-2 and might be involved in nervous system infection. ACE2 is widely expressed throughout the nervous system $^{14-16}$, making neural cells susceptible to infection. Interestingly, ACE2 is expressed in nuclei involved in the central regulation of cardiovascular and respiratory functions, such as the paraventricular nucleus, the nucleus of the tractus solitarius (NTS) and the rostral ventrolateral medulla, as well as in the motor cortex and raphe ${ }^{17}$.

In this Perspective, we briefly summarize our current knowledge regarding the immune response to SARS-CoV-2. We then consider how lung afferent and efferent innervation might crosstalk with the immune system to modulate lung function, thereby critically affecting clinical outcomes following SARS-CoV-2 infection.

\section{The antiviral immune response}

The respiratory tract is a major barrier tissue and is often the first point of contact and entry for harmful substances and pathogens, including viruses. Alveolar epithelial cells are important cellular targets for SARS-CoVs and the avian $\mathrm{H} 5 \mathrm{~N} 1$ virus $^{18,19}$, which elicit a prompt immune response. While attempting to eliminate the virus, this response can compromise respiratory function owing to excessive tissue inflammation, leading to acute respiratory distress syndrome (ARDS).

The first immune cell types to encounter viral antigens in the respiratory tract are the alveolar and interstitial macrophages, which are capable of eliminating many different pathogens from the lungs by phagocytosis. In addition, interstitial macrophages seem to be involved in dampening the immune response in loco after influenza virus infection ${ }^{20,21}$. Dendritic cells are the primary antigen-presenting cells (APCs), and resident mature $\mathrm{MHCII}{ }^{\text {hi }} \mathrm{CD} 11 \mathrm{c}^{\text {hi }}$ respiratory dendritic cells are crucial for the induction of adaptive immune responses. Plasmacytoid dendritic cells are also recognized as major producers of type I interferons during infection and can transport antigens from the infected lung to the draining lymph nodes, where they serve as potent APCs for the induction of virus-specific T cells ${ }^{22,23}$.

Following exposure to APCs in the draining lymph nodes, virus-specific naive T cells undergo a stepwise process of activation, proliferation and differentiation to become effector $\mathrm{T}$ cells, which can migrate to the site of infection and mediate antiviral immune responses. Virus-specific memory $\mathrm{T}$ cells are antigen-experienced 
cells that have acquired the capacity to rapidly implement effector functions and exert antiviral activity. Three main antiviral effector mechanisms have been identified: lysis of infected cells following exocytosis of granules containing perforin and granzyme from cytotoxic T cells, possibly with the cooperation of B cells; tumour necrosis factor (TNF) receptor family-dependent apoptosis of infected cells and phagocytosis following recognition of these cells by antibodies; and production of pro-inflammatory mediators by $\mathrm{T}$ cells in response to encountering virus-infected cells.

\section{Immune responses to SARS-CoV-2}

Clinically, the immune responses induced by SARS-CoV-2 infection consist of two phases. During the initial incubation and in non-severe stages, a specific adaptive immune response is produced to eliminate the virus and abort disease progression. If the protective immune response is impaired, the virus is allowed to replicate and can cause widespread tissue damage. The lungs seem to be the main target, although other organs with high ACE2 expression, such as the intestine, heart, brain and kidney, might also be vulnerable ${ }^{11,24}$. The second phase is usually associated with increased severity of the disease and is characterized by potentially life-threatening lung inflammation ${ }^{25}$ and the appearance of systemic symptoms such as high fever and severe muscle pain.

The damaged cells in the lungs elicit a strong innate immune response, which seems to be largely mediated by pro-inflammatory macrophages and granulocytes ${ }^{26,27}$. This response is thought to be responsible for the cytokine release syndrome or 'cytokine storm' that is believed to contribute to $\mathrm{ARDS}^{25}$. The idea that the cytokine storm is mediated by leukocytes other than $\mathrm{T}$ or $B$ cells is supported by observations of lymphocytopenia and sometimes atrophy of the lymphatic tissues, such as lymph nodes and spleen, in patients with severe COVID-19 (REFS ${ }^{24,26,28-30}$ ). In line with observations in lethal cases of SARS ${ }^{31}$ and MERS $^{32}$, patients infected with SARS-CoV-2 show increased numbers of neutrophils and macrophages in their airways and blood ${ }^{28,33}$.

Levels of inflammatory cytokines such as IL-1, TNF and IL- 6 can be high in the lungs of patients with COVID-19 $\left(\mathrm{REFS}^{1,34}\right)$; therefore, blocking one or more of these cytokines could potentially benefit these individuals by reducing the severity of the cytokine storm and ARDS. Initial attempts to improve COVID-19 outcomes by blocking IL-6 have shown some promise ${ }^{35}$, although the complexity of the immune response to SARS-CoV-2 might limit the efficacy of this approach. A recent study found that in individuals with severe COVID-19, plasma levels of IL- 6 were generally above the normal range but lower than the median values typically reported in $\mathrm{ARDS}^{36}$, suggesting that the inflammatory and cytokine profiles induced by SARS-CoV-2 infection are more complex than previously thought, and that the role of cytokine dysregulation following SARS-CoV-2 infection requires further clarification. Elevated levels of cytokines might have an impact on lung function even when below the cytokine storm threshold because of the unique inflammatory profile associated with COVID-19. In addition, levels of specific cytokines might be elevated predominantly in the lung alveolar microenvironment, contributing to respiratory distress.

Interestingly, with age, macrophage activity declines and pro-inflammatory cytokine levels increase ${ }^{37}$; moreover, naive $\mathrm{T}$ cell production and effector memory $\mathrm{T}$ cell competency decline ${ }^{38,39}$. Together, these phenomena could contribute to worsening of the severity and outcomes of SARS-CoV-2 infection in older individuals, as discussed in more detail below.

\section{Lung innervation and immunity}

The possible role of lung innervation in the regulation of the immune response to SARS-CoV-2 is an important area of investigation. The lungs are highly innervated, receiving sensory, sympathetic and parasympathetic fibres (FIG. 1). Sympathetic innervation of the lungs originates from sympathetic ganglia that arise from the upper thoracic segments of the spinal cord and provide noradrenergic innervation to bronchial blood vessels and submucosal glands. The tenth cranial nerve, or vagus nerve, supplies all parasympathetic and most of the sensory nerve fibres to the airways, although some more minor sensory innervation originates from the T1-T6 dorsal root ganglia.

The vagus nerve divides into the superior laryngeal and recurrent laryngeal nerves, which carry sensory fibres originating in the jugular and nodose ganglia and parasympathetic cholinergic fibres originating in the petrosal ganglion to the trachea and main bronchi. Smaller branches of the vagus nerve innervate the rest of the lower respiratory tract ${ }^{40,41}$.

Stimulation of parasympathetic pathways induces bronchoconstriction, mucus secretion and bronchial vasodilation, mediated by muscarinic M3 receptors ${ }^{42,43}$. Muscarinic M2 receptors are present on presynaptic cholinergic nerve terminals ${ }^{44}$, and activation of these receptors inhibits acetylcholine release and might serve to limit cholinergic bronchoconstriction. Interestingly, parainfluenza virus has been shown to decrease M2 muscarinic receptor function on parasympathetic nerves $^{45}$, and loss of M2 receptor-dependent negative feedback increases acetylcholine release onto airway smooth muscle ${ }^{46}$. Expression of M2 and M3 receptors by lung macrophages has been described ${ }^{47}$, suggesting that acetylcholine could directly influence lung macrophage activity and, thus, inflammation. In addition, cytokines such as IFN $\gamma$, TNF and IL- $1 \beta$ seem to influence both the expression and the activity of $\mathrm{M} 2$ receptors ${ }^{48-50}$. These cytokines are released in response to viral infection and their levels are increased in the lungs of patients infected with SARS-CoV or SARS-CoV-2 (REFS ${ }^{1,51,52}$ ). In line with these findings, virus-induced airway hyperresponsiveness in humans seems to be mediated by the vagus nerve ${ }^{53}$, raising the possibility that the dyspnoea and respiratory failure observed in patients with severe COVID-19 is exacerbated by neuroimmune crosstalk in the lungs.

Most of the afferent sensory fibres arising from the airways run along the vagus nerve and project to the NTS in the medulla. A small population of sensory fibres that innervate the lungs, known as the chemosensitive $\mathrm{C}$-fibres, contain neuropeptides, including calcitonin gene-related peptide (CGRP) and the tachykinins substance $\mathrm{P}$ and neurokinin $\mathrm{A}$. Substance $\mathrm{P}$ and neurokinin A are potent inducers of airway smooth muscle contraction, vasodilation, bronchial oedema and mucus hypersecretion, which are all typical symptoms of inflammatory airway diseases.

A recently discovered class of vagal throat-innervating $\mathrm{P} 2 \mathrm{RY} 1^{+}$neurons has been shown to be involved in protective reflexes elicited by various airway irritants ${ }^{54}$. Although their precise mechanism of action remains elusive, these sensory neurons seem to detect cell mechanical responses mediated by airway stretch receptors such as Piezo2 and initiate defensive reflexes that ensure airway integrity ${ }^{55}$. Dysfunction of laryngeal neurons, which is life-threatening and can cause pulmonary aspiration, dysphagia and choking, might contribute to the respiratory distress observed in patients with COVID-19. Interestingly, 


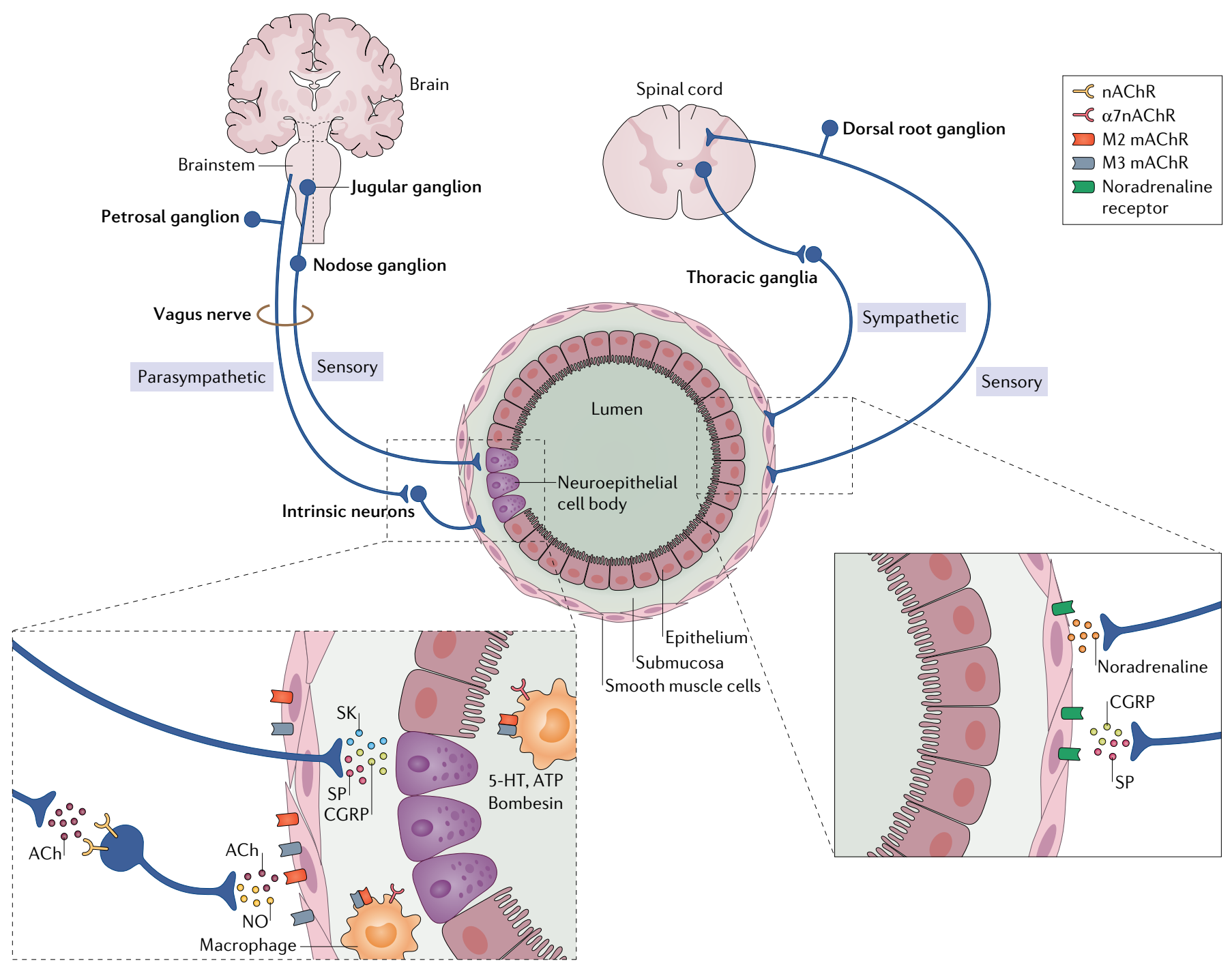

Fig. 1 | Innervation of the respiratory tract. The vagus nerve originates in the brainstem and provides most of the sensory and all of the parasympathetic nerve fibres that innervate the airways, via the jugular and nodose ganglia (sensory) and petrosal ganglion (parasympathetic). A minority of sensory fibres in the lungs originate in the T1-T6 dorsal root ganglia and run in spinal nerves along with sympathetic fibres originating in the thoracic ganglia. The insets show the principal neurotransmitters originating from the fibres that innervate the airways and the target receptors of these neurotransmitters. 5-HT, 5-hydroxytryptamine; ACh, acetylcholine; CGRP, calcitonin gene-related peptide; $\mathrm{mAChR}$, muscarinic $\mathrm{ACh}$ receptor; nAChR, nicotinic ACh receptor; NO, nitric oxide; SK, substance K; SP, substance $P$. altered activation of vagal fibres can lead to many of the symptoms of allergic diseases, such as asthma and rhinitis, and of chronic obstructive pulmonary disease (COPD), including sneezing, coughing, mucus hypersecretion and bronchoconstriction ${ }^{42,56}$.

Another class of sensors that are present in the respiratory tract are the neuroepithelial cell bodies (NEBs). NEBs contain several bioactive mediators, including bombesin, CGRP, 5-hydroxytryptamine and ATP ${ }^{57}$. They form compact structures located within the airway mucosa with their apical surface exposed to the airway lumen and are innervated mostly by vagal afferent fibres ${ }^{58}$. NEBs are multimodal sensors that respond to a range of stimuli in the airway lumen, including hypoxia, hypercapnia, mechanical stretch, nicotine and nociceptive irritant $s^{59,60}$. Hyperplasia of NEBs has been reported in a number of paediatric and adult lung diseases, including viral infections, many of which are associated with symptoms of dyspnoea on presentation ${ }^{57,61}$. The effects of NEB-derived CGRP include bronchoconstriction, vasodilation and modulation of inflammatory responses in the lung ${ }^{62}$. Bombesin, the principal peptide in human NEBs, might also be involved in modulating respiratory function and immune responses in the lungs either by directly inducing bronchoconstriction or by signalling to inflammatory cells $s^{63,64}$.

Importantly, in a number of studies the vagus nerve has been found to have an immunomodulatory (but mainly anti-inflammatory) function ${ }^{65-68}$. The immunomodulatory effects seem to be mediated by three different pathways. The first pathway is the neuroendocrine immune axis that connects the CNS with the intestinal immune system, where pro-inflammatory cytokines (IL-1 $\beta$, IL-6 and TNF) released from the intestinal mucosa activate vagal afferents that terminate in the NTS $^{65}$. The second pathway is the splenic sympathetic anti-inflammatory pathway, in which the vagus nerve stimulates the splenic sympathetic nerve. In this pathway, noradrenaline released at the distal end of the splenic nerve activates $\beta 2$ adrenergic receptors of splenic lymphocytes, which release acetylcholine. In turn, 


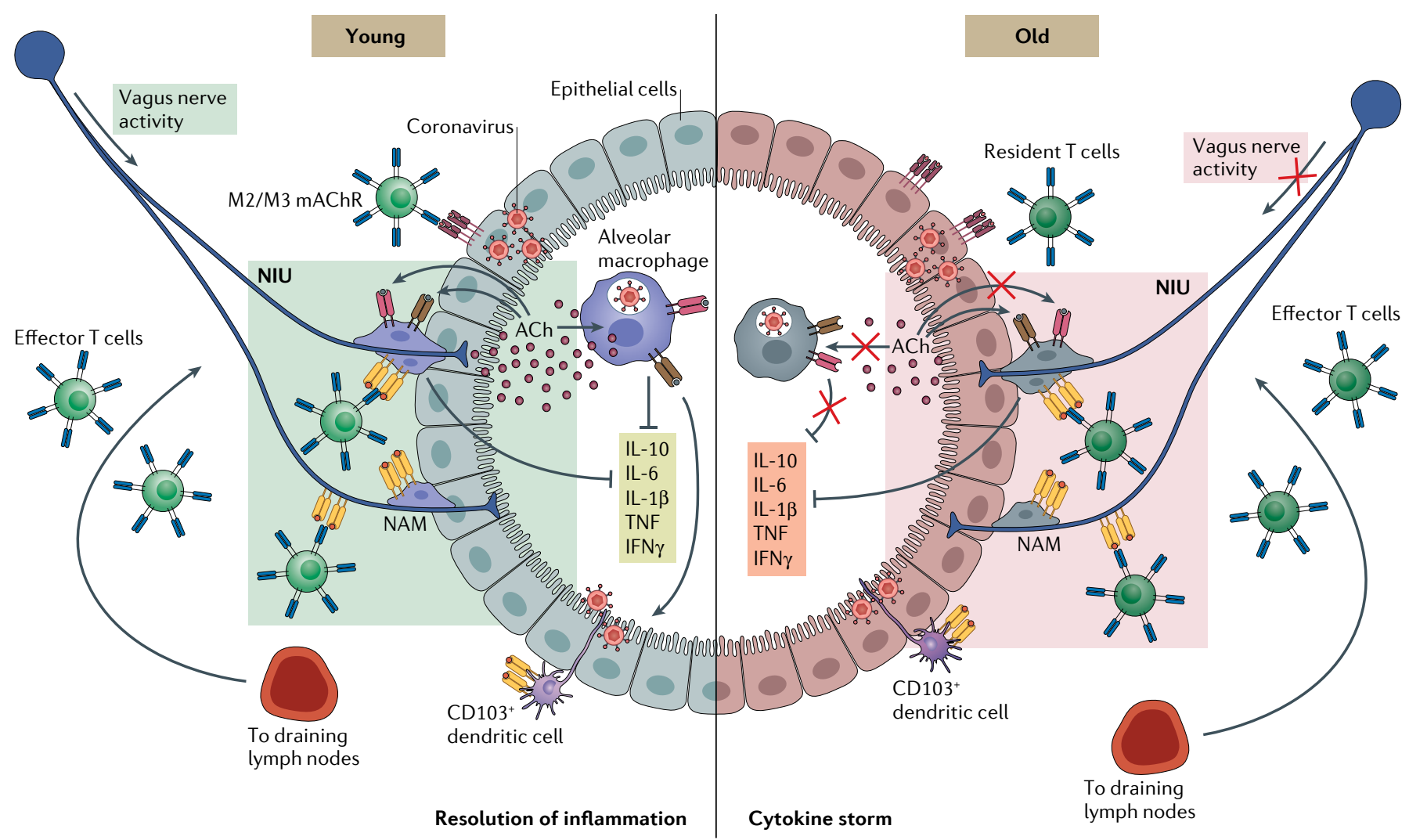

Fig. 2 | The neuroimmune unit. The diagram depicts the proposed constituents of the neuroimmune unit (NIU) and its age-dependent dysfunctional activation. Vagal fibres innervating the airways are in close contact with nerveand airway-associated macrophages (NAMs) and communicate with resident macrophages through the release of neurotransmitters, such as acetylcholine ( $\mathrm{ACh}$ ), and neuropeptides. The macrophages, in turn, modulate the innate immune response and dampen inflammation following events such as SARS-CoV-2 infection. Ageing induces a decline in vagal activity and immune surveillance as well as an increase in the production of pro-inflammatory cytokines. Following viral infection, these cytokines are further produced locally by immune cells, but their levels are potentially reduced by vagus nerve activity, leading to resolution of inflammation. With ageing, decreases in vagal immunomodulatory function and the immune cell response to pathogens, combined with increased levels of pro-inflammatory cytokines, could contribute to the induction of a cytokine storm, leading to respiratory failure and death. mAChR, muscarinic $\mathrm{ACh}$ receptor; TNF, tumour necrosis factor. acetylcholine inhibits the release of TNF by spleen macrophages through $\alpha 7$ nicotinic acetylcholine receptors ( $\alpha 7 \mathrm{nAChRs})$. The third pathway is the cholinergic anti-inflammatory pathway mediated through vagal efferent fibres that synapse onto enteric neurons, which in turn release acetylcholine at the synaptic junction with macrophages ${ }^{65,69}$. As lung macrophages have been shown to express M2 and M3 receptors ${ }^{47}$, acetylcholine release in the airways is likely to contribute to immune modulation and cytokine release in the lung microenvironment.

Nerve- and airway-associated macrophages (NAMs) are a newly discovered interstitial tissue-resident macrophage subset found in close association with neuronal projections in the airways ${ }^{21}$. These cells show distinct transcriptional signatures, including $\mathrm{CD} 169^{+} \mathrm{CD} 11 \mathrm{c}^{-}$and $\mathrm{MHCII}^{\mathrm{hi}}$, as well as high expression of genes involved in communication with nerve cells, such as $C 1 q$ and CX3CR1. Ural et al. showed that
NAMs proliferated robustly following infection with influenza virus in wild-type mice. However, in mice genetically depleted of NAMs, infection induced an exaggerated immune response with excessive production of inflammatory cytokines and immune cell infiltration into tissues ${ }^{21}$. Alveolar macrophages are responsible for direct clearance of viruses, whereas NAMs seem to be involved in moderating inflammation in the lungs during viral infection, in part by functioning as APCs, in addition to playing a possible role in neuroimmune communication. A plausible hypothesis is that these NAMs act in concert with neuronal cells to control inflammation, and that malfunctioning of this system in older or immunocompromised people could contribute to the cytokine storm and ARDS in patients with severe COVID-19 or other respiratory virus infections.

Taken together, the findings described in this section indicate that vagus nerve activity could modulate systemic and local inflammation, probably by acting on tissue-resident immune cells, such as NAMs in the lungs. We have termed this functional association between immune and nerve cells the neuroimmune unit (NIU), which is schematically summarized in FIG. 2.

\section{Ageing and neuroimmunity}

The morbidity due to infectious diseases such as respiratory syncytial virus infection, viral influenza and pneumococcal pneumonia is higher in older individuals than in the younger population, suggesting that the ability to resist infectious diseases and mount protective immune responses declines with age ${ }^{70}$. In addition, ageing is associated with enhanced homeostatic activation of the innate immune system, marked by elevated levels of tissue and circulating pro-inflammatory cytokines such as IFN $\gamma$, IL-1 $\beta$, IL- 6 and $\mathrm{TNF}^{71}$, in the absence of an immunological stimulus a phenomenon termed 'inflammageing. Autophagy, which plays a crucial role in the maintenance of macrophage homeostasis and function, diminishes with ageing, 
resulting in macrophages with reduced phagocytosis and nitrite burst activity in the presence of a markedly increased inflammatory cytokine response ${ }^{72}$. Modulation of autophagy might preserve macrophage function during ageing, therefore reducing the morbidity and mortality associated with inflammageing ${ }^{73}$.

The term 'immunosenescence' refers to the functional decay of immunity with age, with thymic involution being an early sign. The hallmarks of immunosenescence include an inverted CD4 to $\mathrm{CD} 8$ ratio, loss of naive $\mathrm{T}$ cells, increased numbers of terminally differentiated $\mathrm{T}$ cells and oligoclonal expansion of virus-specific $\mathrm{T}_{\text {cells }}{ }^{38,74}$. The activation of natural killer (NK) cells is substantially enhanced in older individuals ${ }^{75}$, and latent cytomegalovirus infection has been shown to contribute to the immunosenescence of $\mathrm{T}$ and $\mathrm{NK}$ cells ${ }^{76,77}$. Importantly, senescent $\mathrm{CD} 8^{+} \mathrm{CD} 28^{-}$effector T cells lack perforin, rendering them inefficient as antigen-specific killers during viral infection $^{78,79}$. However, in certain diseases such as COPD and rheumatoid arthritis, $\mathrm{CD} 8^{+} \mathrm{CD} 28^{-}$cells have been reported to express increased levels of the cytotoxic mediators perforin and granzyme $\mathrm{B}$ and the pro-inflammatory cytokines IFN $\gamma$ and TNF, and can cause substantial tissue damage in an antigen non-specific manner ${ }^{80}$.

Ageing is also associated with changes in autonomic nervous system function, characterized by increases in sympathetic and decreases in parasympathetic nervous activity ${ }^{81,82}$. A study of heart physiology showed that vagal control of heart rate and the response of the vagus nerve to stimulation decrease with age ${ }^{83}$. Furthermore, the responsiveness of muscarinic receptors in the heart, but not in the peripheral vasculature, was shown to decline with age ${ }^{83}$. In line with these findings, vagus nerve stimulation (VNS) can ameliorate age-related alterations in the cardiac baroreflex, heart rate and autonomic responses, and is associated with improved cardiopulmonary outcomes ${ }^{84,85}$.

Ageing is associated with alterations in neuronal activity in multiple systems, and changes in neuropeptide release in the lung mucosa could lead to aberrant pulmonary function and immunological modulation. Age-related disruption of the crosstalk between the nervous and immune systems might lead to impaired immunity, clearance and contractility of the airways and could potentially contribute to the cytokine storm and ARDS in people with severe COVID-19.

\section{Implications for COVID-19}

The high infectivity of SARS-CoV-2 and the severe morbidity associated with SARS-CoV-2 infection, along with our limited understanding of the biology of the virus and a lack of effective treatments or vaccines for COVID-19, has made the COVID-19 outbreak a global emergency. The principal lethal threats for patients with COVID-19 are ARDS and respiratory failure. These complications of SARS-CoV-2 infection are predominantly observed in older $^{86}$ and immunocompromised people ${ }^{86}$ and are mainly due to hyperreactivity and maladaptive responses of the immune system, which is unable to mount a balanced inflammatory response and instead produces a sustained, ineffective and often detrimental reaction known as a cytokine storm. Age-related increases in pro-inflammatory cytokine levels and decreases in the efficiency of immune effector cells, such as macrophages or T cells, could make older and immunocompromised people particularly vulnerable to the virus.

Treatments that target immune cells or pro-inflammatory cytokines have been tested with some degree of success, but the role of neuroimmune crosstalk in the context of infectious and inflammatory diseases, including SARS-CoV-2 infection, has been largely overlooked. Nevertheless, strong evidence exists of a mutual association between nerve and immune cells, with aberrant activity of one cell type affecting the other. In the context of SARS-CoV-2 infection, specific tissueresident macrophages that are involved in modulating inflammation following viral infection are in close contact with vagal fibres innervating the lungs, and this 'neuroimmune synapse' could be one of the keys to controlling aberrant inflammation in patients with severe COVID-19. In addition, in several studies neurological symptoms have been observed in patients with COVID-19 (REFS ${ }^{4,5,13,87}$ ), suggesting a possible role for the nervous system in disease progression.

A feasible clinical approach to COVID-19 could involve the modulation of the pulmonary NIU by VNS, using stimulators that are currently available to treat certain forms of pharmacoresistant epilepsy. VNS has been shown to reduce lipopolysaccharide-induced expression of pro-inflammatory cytokines such as IL- $1 \beta$, IL- 6 and TNF in the brain and spleen ${ }^{68}$. As the vagus nerve extensively innervates the lungs, VNS could potentially exert anti-inflammatory actions on the respiratory tract. Vagal activity has a crucial role in lung physiology by controlling airway smooth muscle tone, vasodilation, mucus secretion and mucociliary clearance, mainly through muscarinic receptor activity $^{88}$. In healthy individuals, muscarinic acetylcholine receptor ( $\mathrm{mAChR}$ ) physiology is in homeostasis; however, following SARS-CoV-2 infection, physiological responses may be disrupted, leading to a maladaptive inflammatory response. This disruption could be especially marked in older and immunocompromised individuals, in whom the vagal tone is typically altered and the ageing-dependent inflammatory response to viral infection is poorly controlled.

The exploration of VNS as a possible treatment for COVID-19 is currently limited by the complexity of vagus nerve anatomy and a lack of knowledge of the optimum stimulation parameters. Development of a tailored neuromodulatory approach would require a detailed investigation of vagus nerve anatomy and activity during lung inflammation (FIG. 3). It is known that the release of acetylcholine by vagal terminations in the airways could be important not only for smooth muscle contraction $^{89}$, mucus secretion ${ }^{90,91}$ and vasodilation in pulmonary vessels ${ }^{92}$ but also to modulate lung macrophage activity and inflammatory responses. A functional role for $\mathrm{mAChRs}$ in immune responses has been demonstrated in lung mast cells and alveolar macrophages. For instance, histamine release from mast cells is blocked by acetylcholine and other $\mathrm{mAChR}$ agonists in human bronchi ${ }^{93,94}$. In support of a macrophage-dependent mechanism, M3 receptor antagonists prevent acetylcholine-induced release of chemotactic cytokines from macrophages ${ }^{95}$.

In addition to muscarinic-dependent inflammation, nAChRs have been suggested to serve as co-receptors for SARS-CoV-2 $\left(\mathrm{REF}^{96}\right)$. The $\mathrm{nAChR}$ pathways that respond to VNS can modulate inflammation via $\alpha 7 \mathrm{nAChR}^{69}$, which is expressed by macrophages. Agonists of $\alpha 7 \mathrm{nAChR}$, including nicotine, have been proven to reduce macrophage-dependent cytokine production and inflammation in animal models of pancreatitis ${ }^{97}$ and peritonitis ${ }^{98}$. Thus, nAChR modulation by SARS-CoV-2 might account for the hyperinflammatory features that are observed in a subgroup of patients with COVID-19. Together, these observations suggest that the use of clinically suitable acetylcholine receptor agonists or antagonists could help dampen the inflammatory response in the lung 

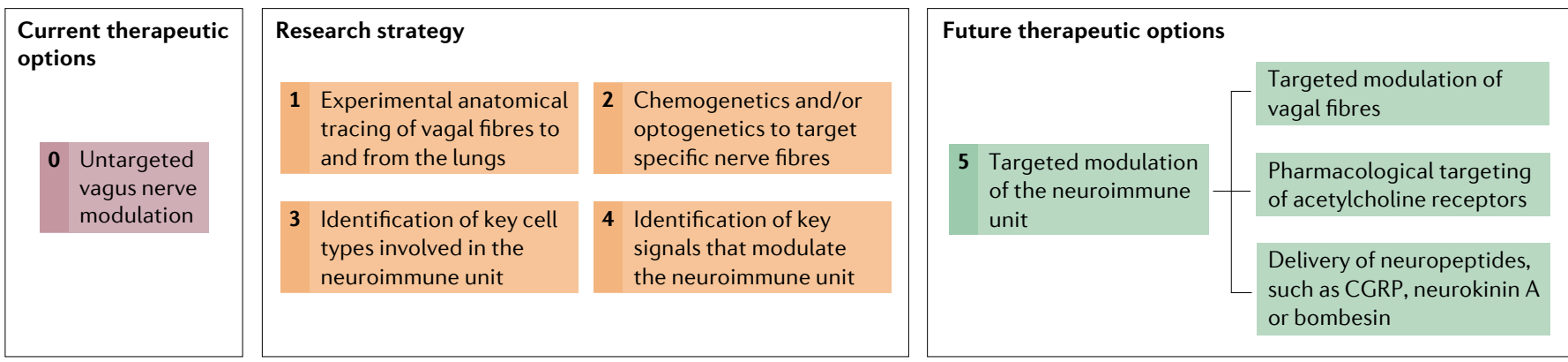

Fig. 3 | Studying the neuroimmune unit: a roadmap. The diagram depicts a stepwise roadmap to increase our understanding of the neuroimmune unit and the potential discovery of pharmacological, genetic and physiological treatments that could be used in the clinic. CGRP, calcitonin gene-related peptide.

microenvironment in the context of SARS-CoV-2 infection.

Further studies are needed to clarify the role and importance of neuroimmune crosstalk both in patients with COVID-19 and in animal models of COVID-19 (FIG. 3). The use of animal models could help uncover the cellular and molecular mechanisms that underlie the mutual relationships between specific nerve fibres and selected immune cell subsets. In experimental models, chemogenetic and optogenetic techniques can be used to manipulate neuronal activity by targeting specific nerve fibres to test how they modulate immunity. Selective immune cell depletion or immunomodulation could provide further insights into neuroimmune communication. In the clinic, the neuroimmune synapses could be visualized by electron microscopy and further analysed by immunohistochemistry in human biopsy or post-mortem tissues. Human sputum or blood samples, as well as lung parenchyma obtained by biopsy, could also be tested for the presence of specific cytokines and cell types.

The presence of ACE2 on neurons makes them susceptible to SARS-CoV-2 infection, which could lead to neuronal damage and aberrant signalling. Afferent and efferent vagal fibres are functionally and molecularly distinct as they convey information in opposite directions, release specific neurotransmitters and express unique combinations of receptors that could communicate with different immune cell subtypes. These fibres comprise a sensory-motor feedback loop that regulates physiological respiratory functions, the disruption of which by viral infection could cause respiratory dysfunction. The vagus nerve could also provide an entry point for the virus to the CNS, including areas of the brain that control respiration, such us the NTS. The NTS is involved in the control of several critical functions besides respiration that might be affected following infection, including cardiac rhythm and food intake regulation. Therefore, vagal infection may result not only in respiratory distress but also in other symptoms such as cardiac dysfunction or loss of appetite. These hypotheses could be tested experimentally using chemogenetic and optogenetic techniques to selectively activate or inhibit afferent or efferent vagal tracts and examine whether this modulation affects immune-dependent and immune-independent respiratory, cardiac and other autonomic functions in health and disease.

In line with our NIU model, therapeutic targeting of transient receptor potential cation channel subfamily V member 1 (TRPV1)-expressing nerve fibres in the lungs, using the TRPV1 agonist resiniferatoxin, has been suggested to modulate inflammatory and immune signalling activity, leading to reduced mortality and better overall outcomes in people with COVID-19 (REF. ${ }^{99}$ ). Although this approach seems promising, several potential downsides need to be considered. TRPV1 is expressed only by a small subset of chemoreceptor fibres (the C-fibres), but the majority of afferent fibres innervating pulmonary structures are carried by vagal or dorsal root ganglion fibres from the upper thoracic segments. In addition, resiniferatoxin is an ultra-potent agonist that binds to and forces the opening of the TRPV1 channel, leading to a sustained increase in intracellular calcium levels ${ }^{100}$. This, in turns, disrupts mitochondrial metabolism and results in permanent neurolysis, making this drug potentially toxic for nerve cells and pulmonary neuroimmune homeostasis.

Another potentially interesting unexplored target is the neuropeptide CGRP. CGRP antagonism could limit bronchoconstriction and vasodilation and limit the inflammatory response ${ }^{62}$, and the recent development and commercialization of anti-CGRP and anti-CGRP receptor monoclonal antibodies for the treatment of migraine might enable fast repurposing for ARDS. Similarly, neurokinin A receptor antagonists, which antagonize the binding of both neurokinin A and the closely related substance $P$, have been developed for clinical use against nausea and vomiting following surgery and chemotherapy ${ }^{101,102}$, and could be repurposed to counteract lung inflammation and bronchoconstriction in people with COVID-19. Nausea and vomiting, which are controlled by the area postrema, NTS and dorsal motor nucleus of the vagus, have also been reported in patients with COVID-19 and could potentially be treated with neurokinin A receptor antagonists.

Additional drugs that specifically modulate the NIU, such as bombesin antagonists, might be developed to improve clinical outcomes in patients with COVID-19 and could also be tested in a broader range of neuroimmune diseases.

\section{Conclusions}

In conclusion, the integrated NIU model that we have proposed suggests novel strategies for the development of targeted and effective treatments that could ameliorate the symptoms and mitigate the life-threatening consequences of SARS-CoV-2 infection. A strategic roadmap for further research and therapeutic development is provided in FIG. 3. In the long term, this model might also provide a new perspective for the discovery of effective therapies for a broad range of inflammatory and infectious diseases and the discovery of potentially novel biological phenomena.

Francesco De Virgiliis $\bowtie$ and Simone Di Giovanni (iD) $\bowtie$

Department of Brain Sciences, Division of Neuroscience, Imperial College London, London, UK.

凶e-mail: francesco.de-virgiliis14@imperial.ac.uk; s.di-giovanni@imperial.ac.uk

https://doi.org/10.1038/s41582-020-0402-y

Published online 25 August 2020 
1. Huang, C. et al. Clinical features of patients infected with 2019 novel coronavirus in Wuhan, China. Lancet 395, 497-506 (2020).

2. Zhou, P. et al. A pneumonia outbreak associated with a new coronavirus of probable bat origin. Nature $\mathbf{5 7 9}$ 270-273 (2020).

3. Butowt, R. \& Bilinska, K. SARS-CoV-2: olfaction, brain infection, and the urgent need for clinical samples allowing earlier virus detection. ACS Chem. Neurosci. 11, 1200-1203 (2020)

4. Yin, R. et al. Concomitant neurological symptoms observed in a patient diagnosed with coronavirus disease 2019. J. Med. Virol. https://doi.org/10.1002/ jmv. 25888 (2020).

5. Mao, L. et al. Neurologic manifestations of hospitalized patients with coronavirus disease 2019 in Wuhan, China. JAMA Neurol. 77, 683-690 (2020).

6. Li, H., Xue, $\mathrm{Q} . \& \mathrm{Xu}, \mathrm{X}$. Involvement of the nervous system in SARS-CoV-2 infection. Neurotox. Res. 38, 1-7 (2020).

7. Rogers, J. P. et al. Psychiatric and neuropsychiatric presentations associated with severe coronavirus infections: a systematic review and meta-analysis with comparison to the COVID-19 pandemic. Lancet Psychiatry 7, 611-627 (2020).

8. De Felice, F. G., Tovar-Moll, F., Moll, J., Munoz, D. P. \& Ferreira, S. T. Severe acute respiratory syndrome coronavirus 2 (SARS-CoV-2) and the central nervous system. Trends Neurosci. 43, 355-357 (2020).

9. Hamming, I. et al. Tissue distribution of ACE2 protein, the functional receptor for SARS coronavirus. A first step in understanding SARS pathogenesis. J. Pathol. 203, 631-637 (2004).

10. Hoffmann, M. et al. SARS-CoV-2 cell entry depends on ACE2 and TMPRSS2 and is blocked by a clinically proven protease inhibitor. Cell 181, 271-280.e8 (2020).

11. Chen, L., Li, X., Chen, M., Feng, Y. \& Xiong, C. The ACE2 expression in human heart indicates new potential mechanism of heart injury among patients infected with SARS-CoV-2. Cardiovasc. Res. 116, 1097-1100 (2020)

12. Saraiva, L. R. et al. Hierarchical deconstruction of mouse olfactory sensory neurons: from whole mucosa to single-cell RNA-seq. Sci. Rep. 5, 18178 (2015).

13. Paybast, S., Emami, A., Koosha, M. \& Baghalha, F. Novel coronavirus disease (COVID-19) and central nervous system complications: what neurologist need to know. Acta Neurol. Taiwan. 29, 24-31 (2020).

14. Elased, K. M., Cunha, T. S., Marcondes, F. K. \& Morris, M. Brain angiotensin-converting enzymes: role of angiotensin-converting enzyme 2 in processing angiotensin II in mice. Exp. Physiol. 93, 655-675 (2008).

15. Ogata, Y. et al. Anti-hypersensitive effect of angiotensin (1-7) on streptozotocin-induced diabetic neuropathic pain in mice. Eur. J. Pain. 23, 739-749 (2019).

16. Deng, Y., Tan, X., Li, M.-L., Wang, W.-Z. \& Wang, Y.-K. Angiotensin-converting enzyme 2 in the rostral ventrolateral medulla regulates cholinergic signaling and cardiovascular and sympathetic responses in hypertensive rats. Neurosci. Bull. 35, 67-78 (2019).

17. Xia, H. \& Lazartigues, E. Angiotensin-converting enzyme 2: central regulator for cardiovascular function. Curr. Hypertens. Rep. 12, 170-175 (2010).

18. Shinya, K. et al. Avian flu: influenza virus receptors in the human airway. Nature 440, 435-436 (2006).

19. van Riel, D. et al. H5N1 virus attachment to lowe respiratory tract. Science 312, 399 (2006).

20. Snelgrove, R. J. et al. A critical function for CD200 in lung immune homeostasis and the severity of influenza infection. Nat. Immunol. 9, 1074-1083 (2008).

21. Ural, B. B. et al. Identification of a nerve-associated lung-resident interstitial macrophage subset with distinct localization and immunoregulatory properties. Sci. Immunol. 5, eaax8756 (2020).

22. Braciale, T. J., Sun, J. \& Kim, T. S. Regulating the adaptive immune response to respiratory virus infection. Nat. Rev. Immunol. 12, 295-305 (2012).

23. Kim, T. S. \& Braciale, T. J. Respiratory dendritic cell subsets differ in their capacity to support the induction of virus-specific cytotoxic CD8+ T cell responses. PLOS ONE 4, e4204 (2009).

24. Shi, Y. et al. COVID-19 infection: the perspectives on immune responses. Cell Death Differ. 27, 1451-1454 (2020).

25. Xu, Z. et al. Pathological findings of COVID-19 associated with acute respiratory distress syndrome. Lancet Respir. Med. 8, 420-422 (2020).
26. Wen, W. et al. Immune cell profiling of COVID-19 patients in the recovery stage by single-cell sequencing. Cell Discov. 6, 31 (2020)

27. Zhang, D. et al. COVID-19 infection induces readily detectable morphological and inflammation-related phenotypic changes in peripheral blood monocytes, the severity of which correlate with patient outcome. Preprint at medRxiv https://europepmc.org/article/ ppr/ppr126980 (2020).

28. Wu, F. et al. A new coronavirus associated with human respiratory disease in China. Nature 579, 265-269 (2020).

29. Qin, C. et al. Dysregulation of immune response in patients with COVID-19 in Wuhan, China. Clin. Infect. Dis. 71, 762-768 (2020).

30. Chen, G. et al. Clinical and immunological features of severe and moderate coronavirus disease 2019. J. Clin. Invest. 130, 2620-2629 (2020).

31. Perlman, S. \& Dandekar, A. A. Immunopathogenesis of coronavirus infections: implications for SARS Nat. Rev. Immunol. 5, 917-927 (2005).

32. Zumla, A., Hui, D. S. \& Perlman, S. Middle East respiratory syndrome. Lancet 386, 995-1007 (2015).

33. Du, Y. et al. Clinical features of 85 fatal cases of COVID-19 from Wuhan. A retrospective observational study. Am. J. Respir. Crit. Care Med. 201, 1372-1379 (2020).

34. Henry, B. M., Santos de Oliveira, M. H., Benoit, S. Plebani, M. \& Lippi, G. Hematologic, biochemical and immune biomarker abnormalities associated with severe illness and mortality in coronavirus disease 2019 (COVID-19): a meta-analysis. Clin. Chem. Lab. Med. 58, 1021-1028 (2020)

35. Gritti, G. et al. IL-6 signalling pathway inactivation with siltuximab in patients with COVID-19 respiratory failure: an observational cohort study. Preprint at medRxiv https://doi.org/10.1101/2020.04.01.20048 $561 v 4$ (2020)

36. Sinha, P., Matthay, M. A. \& Calfee, C. S. Is a "cytokine storm" relevant to COVID-19? JAMA Intern. Med. https://doi.org/10.1001/jamainternmed.2020.3313 (2020).

37. Linehan, E. \& Fitzgerald, D. C. Ageing and the immune system: focus on macrophages. Eur. J. Microbiol. Immunol. 5, 14-24 (2015).

38. Li, M. et al. Age related human $\mathrm{T}$ cell subset evolution and senescence. Immun. Ageing 16, 24 (2019).

39. Rane, S., Hogan, T., Seddon, B. \& Yates, A. J. Age is not just a number: naive $T$ cells increase their ability to persist in the circulation over time. PLoS Biol. 16 e2003949 (2018).

40. Bałuk, P. \& Gabella, G. Innervation of the guinea pig trachea: a quantitative morphological study of intrinsic neurons and extrinsic nerves. J. Comp. Neurol. 285 117-132 (1989).

41. Kummer, W., Fischer, A., Kurkowski, R. \& Heym, C. The sensory and sympathetic innervation of guinea-pig lung and trachea as studied by retrograde neuronal tracing and double-labelling immunohistochemistry. Neuroscience 49, 715-737 (1992)

42. Barnes, P. J. Chronic obstructive pulmonary disease. N. Engl. J. Med. 343, 269-280 (2000).

43. Barnes, P. J. Overview of neural mechanisms in asthma. Pulm. Pharmacol. 8, 151-159 (1995)

44. Patel, H. J. et al. Evidence for prejunctional muscarinic autoreceptors in human and guinea pig trachea. Am. J. Respir Crit Care Med 152, 872-878 (1995)

45. Fryer, A. D. \& Jacoby, D. B. Parainfluenza virus infection damages inhibitory M2 muscarinic receptors on pulmonary parasympathetic nerves in the guinea-pig Br. J. Pharmacol. 102, 267-271 (1991).

46. Fryer, A. D. \& Wills-Karp, M. Dysfunction of M2-muscarinic receptors in pulmonary parasympathetic nerves after antigen challenge. J. Appl. Physiol. 71 2255-2261 (1991)

47. Koarai, A et al. Expression of muscarinic receptors by human macrophages. Eur. Respir. J. 39, 698-704 (2012).

48. Rynko, A. E., Fryer, A. D. \& Jacoby, D. B. Interleukin-1 mediates virus-induced $\mathrm{M} 2$ muscarinic receptor dysfunction and airway hyperreactivity. Am. J. Respir. Cell Mol. Biol 51, 494-501 (2014).

49. Jacoby, D. B., Xiao, H. Q., Lee, N. H., Chan-Li, Y. \& Fryer, A. D. Virus- and interferon-induced loss of inhibitory M2 muscarinic receptor function and gene expression in cultured airway parasympathetic neurons. J. Clin. Invest. 102, 242-248 (1998)

50. Grodzki, A. C. G., Ghogha, A., Mangini, L., Fryer, A. D. $\&$ Lein, P. J. IFN $\gamma$ increases M2 muscarinic receptor expression in cultured sympathetic neurons. Curr. Neurobiol. 2, 23-29 (2011).
51. Yoshikawa, T., Hill, T., Li, K., Peters, C. J. \& Tseng, C.-T. K. Severe acute respiratory syndrome (SARS) coronavirus-induced lung epithelial cytokines exacerbate SARS pathogenesis by modulating intrinsic functions of monocyte-derived macrophages and dendritic cells. J. Virol. 83, 3039-3048 (2009).

52. Conti, P. et al. Induction of pro-inflammatory cytokines (IL-1 and IL-6) and lung inflammation by Coronavirus-19 (COVI-19 or SARS-CoV-2): anti-inflammatory strategies. J. Biol. Regul. Homeost. Agents 34, 327-331 (2020).

53. Empey, D. W., Laitenen, L. A., Jacobs, L., Gold, W. M. \& Nadel, J. A. Mechanisms of bronchial hyperreactivity in normal subjects after upper respiratory tract infection. Am. Rev. Respir. Dis. 113, 131-139 (1976).

54. Prescott, S. L., Umans, B. D., Williams, E. K. Brust, R. D. \& Liberles, S. D. An airway protection program revealed by sweeping genetic control of vagal afferents. Cell 181, 574-589 (2020).

55. Nonomura, K. et al. Piezo2 senses airway stretch and mediates lung inflation-induced apnoea. Nature $\mathbf{5 4 1}$, 176-181 (2017)

56. Barnes, P. J. Mechanisms in COPD: differences from asthma. Chest 117, 10S-14S (2000)

57. Cutz, E., Yeger, H., Pan, J. \& Ito, T. Pulmonary neuroendocrine cell system in health and disease. Curr. Respir. Med. Rev. 4, 174-186 (2008).

58. Adriaensen, D. A., Brouns, I, Van Genechten, J. \& Timmermans, J.-P. Functional morphology of pulmonary neuroepithelial bodies: extremely complex airway receptors. Anat. Rec. A Discov. Mol. Cell. Evol. Biol. 270, 25-40 (2003).

59. Johnson, D. E. \& Georgieff, M. K. Pulmonary neuroendocrine cells. Their secretory products and their potential roles in health and chronic lung disease in infancy. Am. Rev. Respir. Dis. 140, 1807-1812 (1989).

60. Linnoila, R. I. Functional facets of the pulmonary neuroendocrine system. Lab. Invest. 86, 425-444 (2006).

61. Dakhama, A. et al. The role of virus-specific immunoglobulin $\mathrm{E}$ in airway hyperresponsiveness. Am. J. Respir. Crit. Care Med. 170, 952-959 (2004).

62. Springer, J., Geppetti, P., Fischer, A. \& Groneberg, D. A Calcitonin gene-related peptide as inflammatory mediator. Pulm. Pharmacol. Ther. 16, 121-130 (2003).

63. Gonzalez, N., Moody, T. W., Igarashi, H., Ito, T. \& Jensen, R. T. Bombesin-related peptides and their receptors: recent advances in their role in physiology and disease states. Curr. Opin. Endocrinol. Diabetes Obes. 15, 58-64 (2008).

64. Sunday, M. E., Shan, L. \& Subramaniam, M. Immunomodulatory functions of the diffuse neuroendocrine system: implications for bronchopulmonary dysplasia. Endocr. Pathol. 15, 91-106 (2004).

65. Bonaz, B., Sinniger, V. \& Pellissier, S. The vagus nerve in the neuro-immune axis: implications in the pathology of the gastrointestinal tract. Front. Immunol. 8, 1452 (2017).

66. Payne, S. C. et al. Anti-inflammatory effects of abdominal vagus nerve stimulation on experimental intestinal inflammation. Front. Neurosci. 13, 418 (2019).

67. Pavlov, V. A. \& Tracey, K. J. The vagus nerve and the inflammatory reflex - linking immunity and metabolism. Nat. Rev. Endocrinol. 8, 743-754 (2014).

68. Meneses, G. et al. Electric stimulation of the vagus nerve reduced mouse neuroinflammation induced by lipopolysaccharide. J. Inflamm. 13, 33 (2016).

69. Olofsson, P. S. et al. a7 nicotinic acetylcholine receptor ( $a 7 \mathrm{nAChR}$ ) expression in bone marrowderived non-T cells is required for the inflammatory reflex. Mol. Med. 18, 539-543 (2012)

70. Jansen, A. G. S. C., Sanders, E. A. M., Hoes, A. W. van Loon, A. M. \& Hak, E. Influenza- and respiratory syncytial virus-associated mortality and hospitalisations. Eur. Respir. J. 30, 1158-1166 (2008)

71. Ershler, W. B. \& Keller, E. T. Age-associated increased interleukin- 6 gene expression, late-life diseases, and frailty. Annu. Rev. Med. 51, 245-270 (2000).

72. Stranks, A. J. et al. Autophagy controls acquisition of aging features in macrophages. J. Innate Immun. $\mathbf{7}$ 375-391 (2015)

73. Byrne, A. J., Mathie, S. A., Gregory, L. G. \& Lloyd, C. M. Pulmonary macrophages: key players in the innate defence of the airways. Thorax 70, 1189-1196 (2015).

74. Nikolich-Zugich, J. Ageing and life-long maintenance of T-cell subsets in the face of latent persistent infections. Nat. Rev. Immunol. 8, 512-522 (2008). 
75. Hazeldine, J. \& Lord, J. M. The impact of ageing on natural killer cell function and potential consequences for health in older adults. Ageing Res. Rev. 12, 1069-1078 (2013)

76. Oh, S.-J., Lee, J. K. \& Shin, O. S. Aging and the immune system: the impact of immunosenescence on viral infection, immunity and vaccine immunogenicity. Immune Netw. 19, e37 (2019).

77. Pera, A. et al. Immunosenescence: implications for response to infection and vaccination in older people. Maturitas 82, 50-55 (2015)

78. Reuter M. A et al. HIV-specific CD8 ${ }^{+} \mathrm{T}$ cells exhibit reduced and differentially regulated cytolytic activity in lymphoid tissue. Cell Rep. 21, 3458-3470 (2017).

79. Warren, J. A., Clutton, G. \& Goonetilleke, N. Harnessing $\mathrm{CD}^{+} \mathrm{T}$ cells under HIV antiretroviral therapy. Front. Immunol. 10, 291 (2019).

80. Hodge, $G$. $\&$ Hodge, $S$. Steroid resistant $C D 8+C D 28$ null NKT-like pro-inflammatory cytotoxic cells in chronic obstructive pulmonary disease. Front. Immunol. 7 , 617 (2016).

81. Kuo, T. B. et al. Effect of aging on gender differences in neural control of heart rate. Am. J. Physiol. 277, H2233-H2239 (1999).

82. Hotta, H. \& Uchida, S. Aging of the autonomic nervous system and possible improvements in autonomic activity using somatic afferent stimulation. Geriatr. Gerontol. Int. 10, S127-S136 (2010).

83. Kelliher, G. J. \& Conahan, S. T. Changes in vagal activity and response to muscarinic receptor agonists with age. J. Gerontol. 35, 842-849 (1980).

84. Bretherton, B. et al. Effects of transcutaneous vagus nerve stimulation in individuals aged 55 years or above: potential benefits of daily stimulation. Aging 11, 4836-4857 (2019).

85. Yuan, H. \& Silberstein, S. D. Vagus nerve and vagus nerve stimulation, a comprehensive review: part I. Headache 56, 71-78 (2016)

86. Nikolich-Zugich, J. et al. SARS-CoV-2 and COVID-19 in older adults: what we may expect regarding pathogenesis, immune responses, and outcomes. Geroscience 42, 505-514 (2020).
87. Moro, E. et al. The international European Academy of Neurology survey on neurological symptoms in patients with COVID-19 infection. Eur. J. Neurol. https://doi.org/10.1111/ene.14407 (2020).

88. Buels, K. S. \& Fryer, A. D. Muscarinic receptor antagonists: effects on pulmonary function. Handb. Exp. Pharmacol. 208, 317-341 (2012).

89. Cabezas, G. A., Graf, P. D. \& Nadel, J. A. Sympathetic versus parasympathetic nervous regulation of airways in dogs. J. Appl. Physiol. 31, 651-655 (1971).

90. Baker, B., Peatfield, A. C. \& Richardson, P. S Nervous control of mucin secretion into human bronchi. J. Physiol. 365, 297-305 (1985).

91. Ramnarine, S. I., Haddad, E. B., Khawaja, A. M. Mak, J. C. \& Rogers, D. F. On muscarinic control of neurogenic mucus secretion in ferret trachea. J. Physiol. 494, 577-586 (1996).

92. Laitinen, L. A., Laitinen, M. V. \& Widdicombe, J. G. Parasympathetic nervous control of tracheal vascular resistance in the dog. J. Physiol. 385, 135-146 (1987).

93. Reinheimer, T., Baumgärtner, D., Höhle, K. D., Racké, K $\&$ Wessler, I. Acetylcholine via muscarinic receptors inhibits histamine release from human isolated bronchi. Am. J. Respir. Crit. Care Med. 156, 389-395 (1997).

94. Reinheimer, T., Möhlig, T., Zimmermann, S., Höhle, K. D. $\&$ Wessler, I. Muscarinic control of histamine release from airways. Inhibitory M1-receptors in human bronchi but absence in rat trachea. Am. J. Respir. Crit. Care Med. 162, 534-538 (2000).

95. Reinheimer, T. et al. Glucocorticoids mediate reduction of epithelial acetylcholine content in the airways of rats and humans. Eur. J. Pharmacol. 349, 277-284 (1998).

96. Changeux, J.-P., Amoura, Z., Rey, F. \& Miyara, M. A nicotinic hypothesis for Covid-19 with preventive and therapeutic implications. C. R. Biol. 343, 33-39 (2020).

97. van Westerloo, D. J. et al. The vagus nerve and nicotinic receptors modulate experimental pancreatitis severity in mice. Gastroenterology 130, 1822-1830 (2006).
98. van Westerloo, D. J. et al. The cholinergic antiinflammatory pathway regulates the host response during septic peritonitis. J. Infect. Dis. 191, 2138-2148 (2005).

99. Nahama, A., Ramachandran, R., Cisternas, A. F. $\delta \mathrm{Ji}, \mathrm{H}$. The role of afferent pulmonary innervation in poor prognosis of acute respiratory distress syndrome in COVID-19 patients and proposed use of resiniferatoxin (RTX) to improve patient outcomes in advanced disease state: a review. Med. Drug Discov. 5, 100033 (2020).

100. Karai, L. et al. Deletion of vanilloid receptor 1 -expressing primary afferent neurons for pain control. J. Clin. Invest. 113, 1344-1352 (2004).

101. Chen, S. et al. Human substance P receptor binding mode of the antagonist drug aprepitant by NMR and crystallography. Nat. Commun. 10, 638 (2019).

102. Curran, M. P. \& Robinson, D. M. Aprepitant: a review of its use in the prevention of nausea and vomiting. Drugs 69, 1853-1878 (2009).

\section{Acknowledgements}

The authors thank P. Muraro, M. Botto and R. Reynolds for their valuable critical insight and suggestions.

\section{Author contributions}

Both authors contributed to all aspects of the article.

\section{Competing interests}

The authors declare no competing interests.

\section{Peer review information}

Nature Reviews Neurology thanks J. Sellner, F. Chigr and other, anonymous, reviewer(s) for their contribution to the peer review of this work.

Publisher's note

Springer Nature remains neutral with regard to jurisdictional claims in published maps and institutional affiliations.

(C) Springer Nature Limited 2020 